\title{
Mind-Body Parallelism Revisited
}

\author{
Lawrence Greenman ${ }^{*}$
}

Veterans Administration New Jersey Health Care System, 151 Knollcroft Road, Lyons, NJ 07939, USA

\begin{abstract}
This article addresses the links and interactions between the neuroscience parallel line of theories and the parallel line of mental activity and thinking. An argument is made that the most fundamental biological entity, the human genome, provides a pathway of mind-body functional coordination via a double helix metaphor. Brief selections of neuroscience and psychological theories associated with the characteristics of higher thinking are presented. Advantages of tracing the double-helix/genome pathway to mind -body issues are discussed. A modification is proposed to reflect the unique development of speech and language in human beings. Their effects on thinking and communication warrant a unique avenue of investigation.
\end{abstract}

Keywords: Double helix metaphor, higher thinking, language, neuroscience, parallelism, psychology.

\section{INTRODUCTION}

The mind-body problem as it relates to neurobiology and higher thinking processes has thus far not been comprehensively elucidated. Contemporary research efforts have mainly come from neuroscience sources. Nonetheless, neuroscience knowledge has not been able to account for human behavior and capacity for mental abstraction. The concept of parallelism remains useful as a framework for connecting mind-body functions. My intention is to suggest a mindbody metaphor derived from the double helix parallel structure of the genome. To this end, selected neuroscience and psychological hypothesis and theories will be presented as they bear on mind-body issues. A relevant example of a clinical syndrome will be described. The role of speech and language will be considered as a special and distinctive capacity necessary for exploration and communication of human emotions and corresponding effects on behavior and thinking.

\section{THEORETICAL BACKGROUND}

Although parallelism has been supported by individuals as diverse as Hughling Jackson, Sigmund Freud, Oliver Sacks, and others, it connotes a somewhat barren image. As it stands, parallelism leaves out the ways and means of contact between the biological, neuroscience parallel and the parallel line of mental activity, especially higher level thinking. Parallel lines need not be straight. They may be spherical, convoluted, maintain distances between them, and have cross tracts providing pathways for interconnections. The metaphor I propose is represented by dynamic functional activity; an abstraction of neural circuitry projected throughout the mind-body spectrum.

Watson and Crick discovered the double helix model of DNA (deoxyribonucleic acid) consisting of polynuclear chains wound around each other, a spiral of parallel lines. At

*Address correspondence to this author at the 27 Col. Conklin Drive, Stony Point, New York 10980, USA; Tel: 845942 2357;

E-mail: lgreenmanmd@msn.com first glance, it seems unusual to take the most fundamental entity, the genome contained in the double helix and to connect it with psychology and thinking processes. It is indisputable, however, that the constituents of the genome are represented in the receptor mechanisms of the central nervous system (CNS) autonomic system, and the peripheral nervous system. The membranes of nervous system cells are intrinsically involved in the differentiation, development, and functioning of all human biological organ systems. It is precisely these established scientific facts which the genome expresses in receptors of cell membranes. In turn, it provides a potential to investigate any and all dimensions of mindbody activities. Integration of mind-body is accomplished via intercellular and trans-organ communication. The connecting lines are the candidate gene receptors on nerve cell membranes.

The reader will note that the double-helix metaphor does not deviate from the concept of parallelism but is rather an elaboration of a double helix analogy applied to it. I will now describe how this concept fits in with selections from neuroscience theories and hypothesis, and their links to affects and human mentation.

\section{NEUROSCIENCE THEORIES}

Kandel holds the view that the twenty-first century will succeed in providing a "meaningful bridge between molecular mechanisms and mental processes: a meaningful bridge of cognition". He writes that the information carried by a gene is defined by the sequence of bases along the strand. Consecutive triplets of code words are called codons; with some exceptions, each codon specifies an amino acid, and a string of 100 or more codons provides the genetic code for the assembly of a protein chain. The sequence of amino acids in a protein determines how the chain folds and therefore how it assumes the three dimensional structure necessary for its biological activity. Alteration by mutation in only one nucleotide subunit-one base of one codon will be sufficient to alter the amino acid sequence of the protein and thereby alter the protein properties. Internal and external stimuli- 
steps in the development of the brain, hormones, stress, learning, and social interactions alter the aspect of gene regulation by transcriptional regulators acting on mRNA (messenger ribonucleic acid). This is sometimes referred to as epigenetic gene regulation. Stated simply, the regulation of gene expression by social factors makes all bodily functions, including functions of the brain susceptible to social influence. "The information of DNA (deoxyribonucleic acid) is not translated directly into a protein. Rather the sequences of bases that codes for a protein is transcribed into a complimentary strand of RNA (mRNA) because it carries the information for the sequence of amino acids necessary to construct a protein. The mRNA, in turn, is translated into protein. Thus, altered genes give rise to altered mRNA which produces altered proteins". It is fundamental to keep in mind that a cardinal principle of gene expression and modulation is represented by the cell membranes of the nervous system as well as in the cell membranes of all organ systems. Every facet of the individual components under study is immensely complex. Neurotransmitters, receptor physiology, enhancing and inhibitory factors, the intracellular machinery of second messengers, metabolic cycles and molecular events are exquisitely coordinated. Each of the facets is mediated by the expression of genome derived processes. Kandel states, "The overlap between areas recruited for the perception of real as opposed to imagined visual objects is substantial. It is clear that visual imagination uses the same apparatus of visual perception and the same system of visual attention as would be involved if the stimulus were actually presented to his sense organ." He argues that "in the case of consciousness, it is hard to imagine any solution that would not also require an understanding of the large neural networks that underlie cognition, actions, and emotion." All of the senses are intrinsic to perception but the visual sense is usually dominant and is reflected in the relatively large size of the occipital lobes The retina is, of course, an extension of the brain. Visual images of the external world are formed but they also arise from our inner world from memory storage in the nerve cells and they may also be intentionally created by thoughts and fantasies. Kandel states, "Of all the fields in all of science, the problem of perception, action, memory, attention, and consciousness provide us with the greatest evidence for our lack of understanding as well as our greatest challenge" [1].

Clinically, the syndrome of Post Traumatic Stress Disorder serves as an example of imagery interlaced with different organ systems; somatic symptoms, both motor and autonomic, and with changes in behavior. The observed syndrome is manifested by flashbacks, nightmares, diaphoresis, fight or flight dynamics mediated by neurotransmitters and hormones. Additionally, behavioral changes of withdrawal, isolation, and mental aberrations such as dissociations and hallucinations are present as well. An acute episode may be triggered by various sensory stimuli such as visual, auditory, olfactory, and many others that may have been associated with the experienced trauma.

In a commentary on Kandel's contributions, Hymen states, "The implication for psychiatry in Kandel's work and that of others who have worked on brain plasticity is that life experience and indeed all types of learning, including psy- chotherapy, influence thinking, emotion, and behavior by modifying synaptic connections in particular brain circuits. Moreover, as scientists have shown, circuits are shaped over lifetime by multiple complexly interacting factors including genes, illness, injury, experience, context and chance" [2]. Hyman describes the neurobiology of clinical addictions which he believes might serve as a model for the plasticity of the brain in connection with emotional pleasure factors. His model involves the transcription of RNA over a period of several months [3].

Edelman states that the development of individuals is constrained by genes and inheritance and from early life on the neurodevelopment is epigenetic as well. He states, "Neurons extend the branching process in many directions. This branching generates extensive variability in the connective patterns in individuals which creates an immense diverse repertoire of neural circuits. "Neurons that fire together wire together" in the developing individual. Changes occur between the groups that are functional rather than anatomical...[4] Groups of neurons themselves based on an individual's motor, mental, and trans-organ system activities are disposed into maps in the brain that connect and overlap. There occurs a hierarchal synchronization and integration of the maps into a "global mapping" of various maps. A global mapping activity of the cortex of the brain includes connections, for example, with emotional areas of the brain. The formatting of concepts is the product of the brain categorizing its own activities via memory processes and perceptions both internal and external. Edelman's writings integrates perceptual categorization, memory and learning with "qualia" The latter, Edelman states, "constitute the collection of personal or subjective experiences, feelings and sensations that accompany awareness" [5]. He appreciates and describes the interaction of the unconscious on the conscious mind and gives an accounting of the development of concept thinking, Communication is essential for thought (whether with a real or an imaginary interlocutor). We must use and study these capacities in their own right [6]. As speech and language developed facilitating the groups of neurons involved in the communication, patterns are set down within the nervous system. I would like to emphasize that neurons are not only developing within the nervous system but in conjunction with each and every organ system of the body. The differentiation of organ systems is determined by tissue specific genes at critical stages of development interacting with each other to a greater or lesser degree. Damasio describes two hypotheses which are relevant to the thinking process. The somatic marker hypothesis consists of bodily sensations that are juxtaposition to the brain's capacity to image events and thereby modify the way the brain processes eventual future behavior. Somatic markers are derived from visceral and non- visceral body sensations. The body feelings (gut feelings) function as automated somatic signals when an individual is confronted with a problem that may have a negative or positive outcome. Somatic markers probably influence the efficacy of the decision making process. "They are acquired by experience under the control of an internal preference system and under the influence of an external set of circumstances" [7]. 
In considering the relationship between images and the brain, Damasio posits an image space in which dispositional memories can be reconstructed in recall. He proposes that dispositions are held in neuron assembles called, "conversion zones". Conversion zones are hypothesized to consist of a third party neuron assemble which represents cortical and sub-cortical regions of the brain involved in the act of perceiving and responding to brain images and external objects. Damasio's views on the neural basis of consciousness and the development of a biological self, an autobiographical self- imbued with values, feelings and emotions are not unlike Edelman's qualia [8].

Karen Kaplan-Solms and her husband, Mark described the psychoanalytic exploration of about three dozen patients with specific brain lesions. They apply the method of, "dynamic localization" and clinical syndrome analysis. Not only is the disturbed neurological site itself identified, but component parts as well. It involves the detailed psychological analysis of the structure of the disturbance underlying the manifest symptom. A pathway is thereby explored from specific parts of the brain to how those brain structures contribute and in what way they contribute to the complex functional systems underlying various mental and emotional states [9]. The authors conclude, however, "nevertheless, many gaps in our analytical data remain. We have attempted to fill these gaps-as an interim measure-with reasonable references drawn from existing neuropsychological and psychoanalytical knowledge. It goes without saying that all of these inferences must be treated as highly tentative" [10].

Snyder has conducted experiments with the novel transmitter NOS ( nitrous oxide synthase ) The NOS gene encoded, is a protein of 1, 429 amino acids. NO (nitrous oxide) is formed from the amino acid arginine (coded in the gene) via enzymatic action. Snyder states that, "NO may be a major mediator of sexual and aggressive behavior relevant for studies of their biological determinations in humans as well as in mice. For example, in the clinical syndrome of erectile dysfunction of various etiologies it is well known now that NO modulates penile erection via innervations of genital vascular dynamics [11]. The drug sildenafil enhances the effect of NO. No one doubts the role of visual fantasy and the interplay of various sensory perceptions involving sexuality. One can detect here coordinated gene expression across organ systems of genital functions, multi-sensory, and mental activity.

Although some of the neuroscientists discussed above mention emotions, feelings, sensations, etc., they collectively provide relatively little depth into the understanding of human emotions and higher thought processes. I will, therefore, follow with a section on emotions and ideas.

\section{AFFECTS}

Edelman defines the term "qualia" as a collection of subjective experiences, feelings, and sensations that accompany awareness [12]. Damasio states, " All emotions use the body as their theater ( internal milieu, visceral, vestibular, and musculo-skeletal systems, ) but emotions also effect the mode of operation of numerous brain circuits ; the variety of emo- tional responses is responsible for profound changes in both the body landscape and the brain landscape. The collections of these changes constitute the substrate for the neural patterns which eventually becomes feeling of emotion [13]. Arlow quotes Brenner, "Psychologically affect is a sensation of pleasure, unpleasure, or both plus the ideas associated with the sensation. Ideas and sensation together both conscious and unconscious constitute an affect" [14].

The assessment of affects is a standard in clinical mental status examinations. The key is the relationship between the individual's idea and feeling as judged by the clinician. An inappropriate or incompatible affect indicates a dissociation of verbalized thoughts and the perceived emotion that accompanies it as is commonly observed, for example, in schizophrenia.

The above statements may be viewed as having something elementary flowing through feelings, thoughts, affects, and somatic coordination. It is the innervation of multiple organ systems via differentiated receptors determined by the existing genetic make- up. Affective dysfunction, of course, occurs in connection with various pathologies which are known to degrade the cellular integrity of the central nervous system as in, for example, Alzheimer disease which manifests cognitive deficits. A section below will address cognitive neuroscience after a shift in focus to psychological theories.

\section{PSYCHOLOGICAL THEORIES}

Malcolm Pines stated that Wilhelm von Humboldt taught that, "language is the organ of thought and that intellect and speech are one and inseparable. Goldstein, Head, and Schilder argue that understanding, language, its development and its disorders, is inseparable from studying the human being as a communicative animal. This social animal engages in meaningful communication with himself and his fellows and is a listener to him and thereby knows that the other will understand his utterances. The human being as an articulate communicator is inseparable from the cultural matrix of language and behavior. Paul Schilder and Goldstein developed models which comprise intentionalism, unconscious input, synthesis of psychic function, body image concepts, neural networks, and total unitary function [15].

A center piece of psychological models, for me, was achieved through what Freud referred to as "free association" which he believed was the only basis for claiming psychoanalysis as a scientific method. It culminated in the Structural Theory. The id, ego, and super ego comprise a functional entity which embraces basic biological drives of libido and aggression that Freud paralleled with biological, psychosexual stages of development. Corresponding drive derivatives from dynamic unconscious activities persistently influence thought and human behavior. The double helix metaphor represents a functional entity in parallel with a fundamental biological entity. It is based on biological unfolding of the genome projected through coordinated organ systems inherently innervated by the CNS and autonomic nervous systems linked with the development of memory, images, and language. Brenner emphasized the monitoring 
of sexual and aggressive wishes according to a pleasureunpleasure spectrum.--striving for the former and avoiding the latter. He maintains that, consequently, the mind is in continuous intrinsic conflict and attempts to resolve conflict by compromise formation in thought and action [16]. Arlow distinguishes two phases in psychoanalysis. The first phase is based on the analyst's intuitive, subjective response to the patient's free association of verbalized material. The second phase is based on cognition and reason. Most important is the context in which specific material appears. Contiguity usually suggests dynamic relevance, the configurations of the material, the form and sequence in which the associations appear...repetition and convergence of various themes within the organized bodyof associations.... etc. The second phase of the interactive process is based on cognition and the exercise of reason" [17]. It is evident that verbalized language is the essence of the analytic process.

\section{COGNITIVE NEUROSCIENCE}

The merger of psychology and neuroscience is known as cognitive neuroscience.

The two subordinates of cognition are learning and memory. Kandel states "Learning refers to the modifications of behavior by the acquisition of new information; memory refers to the retention of the information". He holds the view that, "alteration in gene expression induced by learning indicates that a major consequence of such alterations is the growth of synaptic connections and the changes in patterns of neural connections".

"The study of learning has revealed new features of synaptic transmission and new cell biological functions of synaptic signaling. For example, different forms of learning recruit different modulating transmitters which act in one of three ways: 1) They activate second messenger kinases that are transported to the nucleus where they initiate processes required for neural growth and long term memory; 2) They mark the specific synapses for capture of the long term process and regulate local protein synthesis for stabilization; and 3) They mediate in ways we are just beginning to understand, attention processes required for information and recall" [18]. As a product of learning language is obligated to hold a storage place within biological organs. The harvesting of memories is influenced by links to emotional centers of the brain such as the amygdala and related structures of the limbic system. These centers are known to influence emotions and psychological attitudes. In the service of applying neuroscience and psychological theories to a clinical venue, I would like to consider two relevant facets of clinical psychiatry. The first is the genetically based helix expression of target receptors in psychopharmacology and secondly, the current demand for evidence based clinical outcomes in clinical studies.

Psychotropic medications may illustrate the double helix metaphor. They influence target receptors by making contact with genetically determined membrane characteristics. A series of 5-7 helix formations which may open or close pores for the passage of chemical substances or to initiate membrane protein units to follow through with a cascade of intra- cellular events. These events effect synaptic synchronization which ultimately leads to neural tracts ending in the brain where further functional coordination occurs. Thereby, an effect is produced on mood, perceptions (hallucinations), thinking (primary process thinking in delusions) which may result in a beneficial effect in patients. A perusal of the psychotropic medications registered in the Physicians' Desk Reference contains sections related to the mechanisms of actions of the drugs which typically states that, "The exact mechanism of action is unknown", followed by possible hypothesis of how they work. There is a bulk of information, however, that has to do with the psychotropic drug effect on receptors of comprehensive biological organ systems; that is, adverse side effects. Clinicians, therefore, have to balance potential benefits with potential side effects unique to individual patients. Comparative drug studies yield statistical evidence in side effects as well as sought after improvements.

Contemporary psychiatry is focused on evidence based clinical practice". A burden that must be borne, however, is that clinical psychiatry is not completely amenable to rigorous scientific methods since it involves human values and subjectivity. Clinical research drug studies are rated with psychological scales which are but a snapshot in time. The subjects in the studies are affected by the vicissitudes of daily life all through the overall study period. The specifics of neuroscience do not readily carry over to clinical reality. That is partially the reason for the complicated statistical analysis of evidence based data yielding statistical significance or trends. Unfortunately, statistical significance is not the same as clinical significance in individual patients.

It seems fair to say that the findings of neuroscience, the body parallel line of mind-body, has not appreciably elucidated the determinants of human motivation, behavior, or the higher thinking processes. Neuroscientists have produced significant biological findings ranging from a variety of lowly creatures to human beings using exquisite biochemical, molecular biological, and high tech imaging techniques. The accolades, however, went to the neuroscientists not the objects or subjects of their investigations. Awards have been granted as a result of the concept formation capabilities of the neuroscientist's higher thought processes.

Speech and language is the pathway to higher thinking and allows for the open ended formation of concepts. It pertains to a capacity for metaphor, symbolism, and abstraction. Edelman states that, "The fundamental triad of higher brain functions is composed of perceptual categorization, memory, and learning... It involves continual motor activity and repeated rehearsal in different contexts" [19]. Einstein believed that his thinking was primarily visual and muscular. He believed as well that it was a "misconception that facts by themselves can and should yield scientific knowledge free from conceptual construction". His famous thought experiments about visualizing a railroad clock from different vantage points on a speeding train were important to his contemplations about time and space. Einstein realized that the concept of time was "rooted unrecognized in the unconscious. " [20] He was referring to an astronomical context 
whereby what one sees is not necessarily happening at the time one sees it. Here then is a case of intentionally created internal imagery to think through perceptual observations. Einstein applied concept formation to work out the General Theory of Relativity based on visual perceptions, memory, and learning (physics and mathematics). Accordingly, there was parallel and interconnected mind-body circuitry going on that culminated in higher thinking processes.

My notion is that the development of speech and language, which is central to bringing about higher order thinking, involves open-ended associative thought processes unique to the human species and represents distinctive thinking capabilities. Trans-organ system receptor mechanisms are driven by the myriad expressions of the human genome. It seems appropriate to conceive of the genome represented by candidate genes of neuron receptors being (metaphorically) projected throughout total human functioning.

My notion is that the development of speech and language, which is central to bringing about higher order thinking, involves open-ended associative thought processes unique to the human species and represents distinctive thinking capabilities. Trans-organ system receptor mechanisms are driven by the myriad expressions of the human genome. It seems appropriate to conceive of the genome represented by candidate genes of neuron receptors being (metaphorically) projected throughout total human functioning.

\section{DISCUSSION}

The mind-body, double-helix metaphor provides a matrix of communication between mind-body parallel lines via a basic scientific entity. The genome on the double helix is omnipotent in the expression of billions of combinations across neurological developmental differentiation of organ systems. It allows for the epigenetic functional integration of unconscious, conscious, central nervous system, and autonomic nervous system potential for plasticity. Importantly, the double helix metaphor does not contradict established neuroscience findings or observed human clinical behavior.

No animal models exist for higher level thinking associated with complex emotions, language and speech. Investigations involving CNS lesions in humans have constraints in somatic and mental areas. The importance of the human instrument in the person of the psychoanalyst interacting with another human being in the analytic dyad using language and speech has singular value and the psychoanalytic method of free association developed by Freud warrants inclusion in the broad neuroscience enterprise pertaining to mind-body issues. The reason for it is compelling. It has yielded more knowledge and understanding about human thinking, motivation and behavior than all of the neuroscience data and theories put together, Evidence for psychoanalytic theory is its capacity to explain human behavior and mental mechanisms in clinical settings and in everyday life. Additionally, its tenets have permeated our social and cultural institutions such as education, child rearing, interpersonal, and social structures. Neuroscience has acknowledged the major contributions of psychoanalytic theory but tend to think of psychoanalysis as an exhausted method of exploration of the human psyche. It seems to turn rather to the cognitive sciences. The unique development of speech, language and higher thought processes, however, requires a special setting for the investigation of the dynamic unconscious and human emotions. That setting utilizes the free association, scientific method carried out in academic and clinical psychoanalysis. Academic psychology does not deal with the dynamic unconscious and neither does neuroscience. Psychoanalytic exploration of the human psyche is one of the pathways to understand unique human capacities. As neuroscience instructs us, humankind shapes its social-cultural environment. In turn, the plasticity of the genetic expression of the CNS is such that human beings, as they develop throughout life, are shaped by those socio-cultural factors.

\section{CONCLUSION}

A metaphor of mind-body integration has been presented not only for its ability to explain neuroscience information but rather for its ability to have the findings of neuroscience fit into it. A metaphor, the double helix, has been suggested as a functional entity to represent dynamic interactions between mind-body parallels. The complicated interconnections are derived from the human genome with its expression of genes in membranes of the nervous system and organ systems that are the preoccupation of neuroscience. Neuroscience, however, has brought us up to but not into the human drama. The free association method of psychoanalytic investigation warrants inclusion in the common enterprise to understand human emotions and behavior. I don't believe the mind-body, double-helix metaphor will lead investigators astray and I cherish the hope that it will be of value to them. In addition, it may provide a serviceable paradigm for clinicians.

\section{ACKNOWLEDGEMENTS}

The author would like to thank Robert Kovacs and James Delo for their assistance in the preparation of the manuscript and Ron Chase, MD for his thoughtful comments. I am grateful to Maureen Kaune, M, D. for suggestions which are reflected in the content of the article.

\section{CONFLICT OF INTEREST}

Declared none.

\section{REFERENCES}

[1] Kandel, Eric R. Psychiatry, psychoanalysis, and the new biology of mind. Washington DC: American Psychiatric Publishing Inc. 2005; pp. 341-71.

[2] Hymen SE. In: Kandel, Eric R, Eds. Psychiatry, psychoanalysis, and the new biology of mind. Washington DC: American Psychiatric Publishing, Inc. 2005

[3] Hymen SE, Nestler EJ. Initiation on Adaptation: A paradigm for understanding psychotropic drug reaction. Am J Psychiatry 1996; 153-62.

[4] Edelman GM, Tonino G. In: A universe of consciousness: How matter becomes imagination. New York, NY: Basic Books 2000; pp. 79-92.

[5] Edelman GM. In: Bright air brilliant fire: On the Matter of the mind. New York, NY: Basic Books 1992; pp. 111-23.

[6] Edelman GM. In: Bright air, Brilliant fire: On the Matter of the mind. NY: Basic Books 1992; pp. 173-5.

[7] Damasio AR. In: Descartes' error: emotion, reason, and the human brain. New York, NY: GP Putnam 1994; pp. 165-201. 
[8] Damasio AR. In: The feeling of what happens: Body. emotions, and the making of consciousness. New York, NY: Harcourt Brace 1999; pp. 195-233.

[9] Kaplan- Solms K, Solms, M. In: Clinical studies in neuropsychoanalysis: Introduction to a depth neuropsychology. London: Karnac Books 2000; pp. 200-39.

[10] Kaplan-Solms K, Solms, M. In: Clinical studies in neuropsychoanalysis: introduction to a depth neuropsychology. London: Karnac Books 2000.

[11] Snyder S. In: Science and Psychiatry: Groundbreaking discoveries in molecular neuroscience. Washington DC, American Psychiatric Publishing Inc. 2008; pp. 277-87.

[12] Edelman GM. In: Bright air, Brilliant fire: On the matter of mind. New York, NY: Basic Books1992; p. 14.

[13] Damasio AR. In: The feeling of what happens: Body and emotion in the making of consciousness. New York, NY: Harcourt Brace 1999; pp. 51-2.
[14] Arlow J. In: Psychoanalysis: Clinical theory and practice. Madison, CT: International Universities Press 1991; pp. 257-78.

[15] Pines M. In: Freud and the neurosciences. Vienna, Austria: Austrian Academy of Sciences Press 1998.

[16] Brenner C. Conflict, compromise, and the structural theory. Psychoanal Q 2002; 71:407-8

[17] Arlow J. In: Psychoanalysis: Clinical theory and practice. NY: International Universities Press 1991; pp. 277-88.

[18] Kandel, Eric R. In: Psychiatry, psychoanalysis, and the new biology of mind. Washington DC: American Psychiatric Publishing Inc. 2005; pp. 341-67.

[19] Edelman GM. In: Bright air, brilliant fire: On the matter of the Mind. New York, NY: Basic Books 1992; p. 100.

[20] Einstein A. In: Sclilpp P, Ed. Autobiographical notes. La Salle, Illinois: Open Court Publishing Co. 1999.

This is an open access article licensed under the terms of the Creative Commons Attribution Non-Commercial License (http:/creativecommons.org/licenses/by-nc/ 3.0/) which permits unrestricted, non-commercial use, distribution and reproduction in any medium, provided the work is properly cited. 